\title{
Influence of tumour size on the efficacy of targeted alpha therapy with ${ }^{213} \mathrm{Bi}-\left[\mathrm{DOTA}{ }^{0}, \mathrm{Tyr}^{3}\right]$-octreotate
}

Ho Sze Chan ${ }^{1 *}$, Mark W. Konijnenberg ${ }^{1}$, Erik de Blois ${ }^{1}$, Stuart Koelewijn ${ }^{1}$, Richard P. Baum ${ }^{4}$, Alfred Morgenstern ${ }^{3}$, Frank Bruchertseifer ${ }^{3}$, Wouter A. Breeman ${ }^{1}$ and Marion de Jong ${ }^{1,2}$

\begin{abstract}
Background: Targeted alpha therapy has been postulated to have great potential for the treatment of small clusters of tumour cells as well as small metastases. ${ }^{213}$ Bismuth, an a-emitter with a half-life of 46 min, has shown to be effective in preclinical as well as in clinical applications. In this study, we evaluated whether ${ }^{213} \mathrm{Bi}^{-\left[D O T A^{0}\right.}$, $\mathrm{Tyr}^{3}$ ]-octreotate ( ${ }^{213} \mathrm{Bi}$-DOTATATE), a ${ }^{213}$ Bi-labelled somatostatin analogue with high affinity for somatostatin receptor subtype $2\left(S S T R_{2}\right)$, is suitable for the treatment of larger neuroendocrine tumours overexpressing SSTR in comparison to its effectiveness for smaller tumours. We performed a preclinical targeted radionuclide therapy study with ${ }^{213}$ Bi-DOTATATE in animals bearing tumours of different sizes $\left(50\right.$ and $200 \mathrm{~mm}^{3}$ ) using two tumour models: H69 (human small cell lung carcinoma) and CA20948 (rat pancreatic tumour).
\end{abstract}

Methods: Pharmacokinetics was determined for calculation of dosimetry in organs and tumours. H69- or CA20948-xenografted mice with tumour volumes of approximately $120 \mathrm{~mm}^{3}$ were euthanized at 10, 30, 60 and 120 min post injection of a single dose of ${ }^{213}$ Bi-DOTATATE $(1.5-4.8 \mathrm{MBq})$. To investigate the therapeutic efficacy of ${ }^{213}$ Bi-DOTATATE, xenografted $\mathrm{H} 69$ and CA20948 tumour-bearing mice with tumour sizes of 50 and $200 \mathrm{~mm}^{3}$ were administered daily with a therapeutic dose of ${ }^{213}$ Bi-DOTATATE $(0.3 \mathrm{nmol}, 2-4 \mathrm{MBq})$ for three consecutive days. The animals were followed for 90 days after treatment. At day 90, mice were injected with $25 \mathrm{MBq}^{99 \mathrm{~m}} \mathrm{Tc}$-DMSA and imaged by SPECT/CT to investigate possible renal dysfunction due to ${ }^{213}$ Bi-DOTATATE treatment.

Results: Higher tumour uptakes were found in CA20948 tumour-bearing animals compared to those in H69 tumour-bearing mice with the highest tumour uptake of $19.6 \pm 6.6 \% \mathrm{IA} / \mathrm{g}$ in CA20948 tumour-bearing animals, while for H69 tumour-bearing mice, the highest tumour uptake was found to be $9.8 \pm 2.4 \% \mathrm{lA} / \mathrm{g}$. Nevertheless, as the anti-tumour effect was more pronounced in $\mathrm{H} 69$ tumour-bearing mice, the survival rate was higher. Furthermore, in the small tumour groups, no regrowth of tumour was found in two H69 tumour-bearing mice and in one of the CA20948 tumour-bearing mice. No renal dysfunction was observed in ${ }^{213}$ Bi-DOTATATE-treated mice after the doses were applied.

Conclusions: ${ }^{213}$ Bi-DOTATATE demonstrated a great therapeutic effect in both small and larger tumour lesions. Higher probability for stable disease was found in animals with small tumours. ${ }^{213}$ Bi-DOTATATE was effective in different neuroendocrine (H69 and CA20948) tumour models with overexpression of SSTR 2 in mice.

\footnotetext{
* Correspondence: h.s.chan1982@gmail.com

${ }^{1}$ Department of Nuclear Medicine, Erasmus Medical Center, Rotterdam, The

Netherlands

Full list of author information is available at the end of the article
} 


\section{Background}

Peptide receptor radionuclide therapy (PRRT) is an effective treatment option for patients with an inoperable neuroendocrine tumour (NET) or with metastatic disease $[1,2]$. Approximately $80 \%$ of patients with NET show great anti-tumour response upon PRRT. The most commonly used radiolabelled peptides for treatment in NET are $\left.{ }^{177} \mathrm{Lu}_{-\left[\mathrm{DOTA}^{0},\right.}, \mathrm{Tyr}^{3}\right]$ - octreotate $\left({ }^{177} \mathrm{Lu}-\mathrm{DOTATATE}\right)$ and ${ }^{90} \mathrm{Y}$-[DOTA $\left.{ }^{0}, \mathrm{Tyr}^{3}\right]$ - octreotide $\left({ }^{90} \mathrm{Y}\right.$-DOTATOC). ${ }^{90} \mathrm{Y}$ and ${ }^{177} \mathrm{Lu}$ are $\beta$-particles emitting radionuclides, with a maximum tissue penetration of 12 and $2 \mathrm{~mm}$, respectively. For treatment of small tumour clusters and metastases, $\beta$-emitters may lack efficacy due to the relatively large tissue penetration and low linear energy transfer (LET). Targeted alpha therapy (TAT) with $\alpha$-emitters has been described to be more suitable for these purposes, since the path length in tissue of $\alpha$-particles is relatively short (in tissue $50-100 \mu \mathrm{m}$ ) compared to that of $\beta$-particles. Moreover, $\alpha$-particles have a much higher LET $(\sim 100 \mathrm{keV} / \mu \mathrm{m})$ and are therefore more powerful than $\beta$-particles $(0.2 \mathrm{keV} / \mu \mathrm{m})$ for therapeutic applications [3]. Bismuth-213 $\left({ }^{213} \mathrm{Bi}\right)$, an $\alpha$-emitter with a $T_{1 /}$ 2 of $45.6 \mathrm{~min}$, has already been proven to be effective in TAT [4-7]. Recently, a clinical trial showed a promising anti-tumour effect in patients with progressive advanced neuroendocrine liver metastasis refractory to ${ }^{90} \mathrm{Y}$-DOTATOC or ${ }^{177} \mathrm{Lu}$-DOTATOC treatment after treatment with ${ }^{213} \mathrm{Bi}$-DOTATOC [8]. TAT with ${ }^{213} \mathrm{Bi}$-DOTATOC was able to overcome the resistance against $\beta$ radiation in patients with different neuroendocrine tumours, in metastasis and in primary tumours, resulting in a high number of long-lasting anti-tumour responses.

The objective of this study was to investigate the therapeutic efficacy of ${ }^{213} \mathrm{Bi}$-DOTATATE in tumours of different sizes in mice (small vs large). Two different tumour models with somatostatin receptor subtype $2\left(\mathrm{SSTR}_{2}\right)$ expression were used: CA20948 (rat pancreatic tumour) and H69 (human small cell lung carcinoma). Both tumours have relatively high $\mathrm{SSTR}_{2}$ expression, and both have often been applied as a model in preclinical studies of PRRT in NET [9-11]. The radiation sensitivity of CA20948 for low-LET radiation has been determined yielding $\alpha / \beta=$ 8.3 Gy [12]. The H69 cell line shows a higher radiation sensitivity $\alpha / \beta=2.3$ Gy [13]. Radiation damage by the high-LET $\alpha$ radiation from ${ }^{213} \mathrm{Bi}$ is expected to follow a linear (-exponential) curve which was comparable for both cell lines.

For PRRT, the dose-limiting organs are bone marrow and kidneys. ${ }^{213} \mathrm{Bi}$-DOTATATE shows accumulation in the kidneys, resulting in high radiation doses to the kidneys; in this study, we therefore determined the safe dose of radioactivity administered for TAT in this mouse model. Renal function was evaluated after TAT by SPECT/CT at different time points post treatment using ${ }^{99} \mathrm{~m}$ Tc-DMSA (dimercaptosuccinic acid), a renal marker for tubular damage.

\section{Methods}

${ }^{213}$ Bi-eluate

A ${ }^{213} \mathrm{Bi} /{ }^{225} \mathrm{Ac}$ generator $(222 \mathrm{MBq}$ ) was provided by the Institute for Transuranium Elements (Karlsruhe, Germany). Prior to elution, the generator was rinsed using $0.01 \mathrm{M} \mathrm{HCl}(3 \mathrm{~mL})$ and eluted using $0.1 \mathrm{M} / 0.1 \mathrm{M}$ $\mathrm{HCl} / \mathrm{NaI}(0.6 \mathrm{~mL})$. After elution, the generator was rinsed with $0.01 \mathrm{M} \mathrm{HCl}$ and stored in $0.01 \mathrm{M} \mathrm{HCl}$ [4]. The activity of ${ }^{213} \mathrm{Bi}$ was determined using a germanium detector (MetorX, Genie 2000 software, Canberra) [14].

\section{${ }^{213} \mathrm{Bi}$-DOTATATE labelling}

Eluate containing ${ }^{213} \mathrm{Bi}(0.1 \mathrm{M} / 0.1 \mathrm{M}$ in $\mathrm{HCl} / \mathrm{NaI})$ was added to a solution containing $7.0 \mathrm{nmol}$ DOTATATE, $0.15 \mathrm{M}$ TRIS buffer and $2.6 \mathrm{mM}$ ascorbic acid in a total reaction volume of $800 \mu \mathrm{L}$. The reaction mixture was incubated for $5 \mathrm{~min}$ at $95{ }^{\circ} \mathrm{C}$ followed by $5 \mathrm{~min}$ at room temperature. To chelate any unbound/free ${ }^{213} \mathrm{Bi}, 50 \mathrm{nmol}$ DTPA (Sigma Aldrich, Zwijndrecht, The Netherlands) was added (manuscript submitted). Analysis by instant thin-layer chromatography (ITLC, Varian) and reversephase high-performance liquid chromatography (HPLC, 2695 separation module, Alliance, Waters, Etten-Leur, The Netherlands) of the labelled peptide was performed to determine the incorporation yield (\%) and radiochemical purity (RCP) expressed as percentage of labelled peptide of interest compared to other detectable compounds [15]. The whole labelling procedure took on average $20 \mathrm{~min}$ to produce a ready-to-inject vial.

\section{${ }^{99 m}$ Tc-DMSA labelling}

The ${ }^{99 \mathrm{~m}}$ Tc-DMSA kit was purchased from Mallinckrodt (Petten, the Netherlands) and labelled according to the indicated procedure.

\section{Cell culture}

The rat pancreatic tumour cell line CA20948 (derived from a rat pancreas tumour at our institute) with high $\mathrm{SSTR}_{2}$ expression was cultured in DMEM (Gibco, Life Technologies) supplemented with $10 \%$ heat-inactivated fetal bovine serum. H69 tumour cells, human small cell lung carcinoma cells with $\mathrm{SSTR}_{2}$ expression (American Tissue Culture Collection, Wesel, Germany), were cultured in RPMI 1640 (Gibco, Life Technologies) supplemented with $10 \%$ heat-inactivated fetal bovine serum and $50 \mathrm{IU} / \mathrm{mL}$ penicillin/streptomycin. All cells were cultured at $37{ }^{\circ} \mathrm{C}$ in a $5 \% \mathrm{CO}_{2} /$ humidified air atmosphere. 


\section{Animal models}

All animal studies were in agreement with the Animal Welfare Committee requirements of Erasmus $\mathrm{MC}$ and conducted in accordance with accepted guidelines. Male 6-8-week-old BALB/c (nu/nu) nude mice were obtained from Charles River (Kißlegg, Germany). Subcutaneous tumours were established in mice by inoculation of approximately $5 \times 10^{6}$ cells (CA20948 or H69), suspended in $200 \mu \mathrm{L}$ sterile culture medium Hanks' balanced salt solution (HBSS) in the case of CA20948 cells. Three days after inoculation of CA20948 cells, a tumour with a tumour size $\sim 50 \mathrm{~mm}^{3}$ (used for the "smaller" tumoursize group) was reached, and 2 weeks after inoculation, a tumour size $\sim 200 \mathrm{~mm}^{3}$ (used for the "larger" tumoursize group) was reached. For H69 cells, $200 \mu \mathrm{L}$, containing 1/3 Matrigel and 2/3 HBSS medium (Gibco, Life Technologies), was used. The cells were injected subcutaneously (s.c.) into the right flank of the mice. One week after inoculation of tumour cells, tumours with a volume of $\sim 50 \mathrm{~mm}^{3}$ (used for the smaller tumour-size group) could be detected, and after 3 weeks, tumour volumes reached $\sim 200 \mathrm{~mm}^{3}$ (used for the larger tumoursize group). Tumour volumes were estimated using a calliper.

\section{Pharmacokinetics of ${ }^{213} \mathrm{Bi}$-DOTATATE as function of time in different tumour models}

When tumour volumes reached $\sim 120 \mathrm{~mm}^{3}$, animals were divided into five groups with seven animals in each group for both H69 and CA20948 tumour-bearing animals. Four groups of mice received a single intravenous (i.v.) injection of ${ }^{213} \mathrm{Bi}$-DOTATATE $(200 \mu \mathrm{L})$ via the tail vein, $3.8 \pm 0.6 \mathrm{MBq} / 0.3 \mathrm{nmol} / 200 \mu \mathrm{L}$ for $\mathrm{H} 69$ tumourbearing animals and $2.1 \pm 0.2 \mathrm{MBq} / 0.3 \mathrm{nmol} / 200 \mu \mathrm{L}$ for CA20948 tumour-bearing animals. At 10, 30, 60 and $120 \mathrm{~min}$ post injection (p.i.), animals in the four groups were euthanized and tissues and organs of interest (including tumour, kidney, pancreas, spleen, liver, muscle, blood, femur, femur marrow and adrenals) were collected and counted using a gamma counter (Wallac Wizard 3, PerkinElmer, USA). Control animals received a single injection of ${ }^{213} \mathrm{Bi}$-DOTATATE plus an excess of DOTATATE $\left(10^{-6} \mathrm{M}\right)$ i.v. via the tail vein (blocking dose) to determine the non-receptor-specific binding of ${ }^{213} \mathrm{Bi}$-DOTATATE. The animals of the control group were euthanized $60 \mathrm{~min}$ p.i.; tissues and organs of interest were harvested and counted using a gamma counter. Data was expressed in mean percentage of total injected radioactivity per gram tissue $(\% \mathrm{IA} / \mathrm{g}) \pm \mathrm{SD}$.

\section{Targeted alpha therapy}

Tumour cells were inoculated into the right flank of the mice, and the tumours were allowed to grow until tumour volumes of approximately 50 and $200 \mathrm{~mm}^{3}$ were reached, respectively. On the day of treatment ( $T=0$ days), the animals (H69-bearing animals: small tumour $n=10$, large tumour $n=7$; CA20948-bearing animals: small tumour $n=8$, large tumour $n=5$ ) were injected i.v. via the tail vein with ${ }^{213} \mathrm{Bi}$-DOTATATE (2-4 MBq/0.3 nmol/ $200 \mu \mathrm{L})$ for three consecutive days. The control group (H69-bearing animals: small tumour $n=10$, large tumour $n=9$; CA20948-bearing animals: small tumour $n=5$, large tumour $n=5$ ) received i.v. via the tail vein three times $0.3 \mathrm{nmol} / 200 \mu \mathrm{L}$ of unlabelled DOTATATE for three consecutive days.

All animals were followed for 90 days after treatment. The behaviour and the health status of the animals were observed daily, and the weight of the animals and the tumour volumes were monitored twice a week.

\section{Determination of renal function by ${ }^{99 m}$ Tc-DMSA imaging}

Renal function imaging was performed by a four-headed multi-pinhole SPECT/CT camera (Bioscan Inc., Washington D.C., USA). For ${ }^{99 \mathrm{~m}}$ Tc imaging, an energy peak at $140 \mathrm{keV}$ was selected with an energy window width \pm $10 \%$. A nine-pinhole aperture (diameter of each pinhole $1.4 \mathrm{~mm}$ ) was used on each camera head. For the CT topogram, an axial length of $27 \mathrm{~mm}$ was used to cover the renal region. Animals were imaged under anaesthesia by isoflurane and $\mathrm{O}_{2}$. The body temperature of the animals was maintained using a heated bed. Renal imaging of $\mathrm{H} 69$ (control $n=4,{ }^{213} \mathrm{Bi}$-DOTATATE treated $n$ $=8$ ) and CA20948 tumour-bearing animals (control $n=2$, ${ }^{213} \mathrm{Bi}$-DOTATATE treated $n=4$ ) was performed $2 \mathrm{~h}$ after i.p. administration of ${ }^{99 m}$ Tc-DMSA $(25 \mathrm{MBq}) .{ }^{99 m} \mathrm{Tc}-$ DMSA images were acquired for $12 \min (20$ projections, $60 \mathrm{~s} /$ projection) [16].

\section{Absorbed dose in organs}

The absorbed radiation dose (defined as the mean absorbed dose in Gy) in organs and tumours was estimated from the results obtained in the biodistribution studies as described by Konijnenberg et al. [17] in a spherical geometry using Monte Carlo code MCNP5 (version 1.4) for the electrons and $\gamma$-rays [18] and MCNPX (version 2.5) [19] for the $\alpha$-particles, all energy emission spectra were taken from ICRP database [20], and particle histories of $1 \times 10^{7}$ was used for simulation to reduce the variation $(<5 \%)$ in the data. The mean absorbed energies for the $10 \mathrm{MBq}^{213} \mathrm{Bi}$-DOTATATE injection were calculated for spheres of 1, 10, 100, 200, 300 and $500 \mathrm{mg}$ containing tissue with a mass density of $1 \mathrm{~g} / \mathrm{mL}$ to determine the spherical node $S$-values. A homogeneous activity distribution over the sphere was assumed. The absorbed dose $D_{i}$ was calculated for each volume $i$ according to the MIRD schema: 


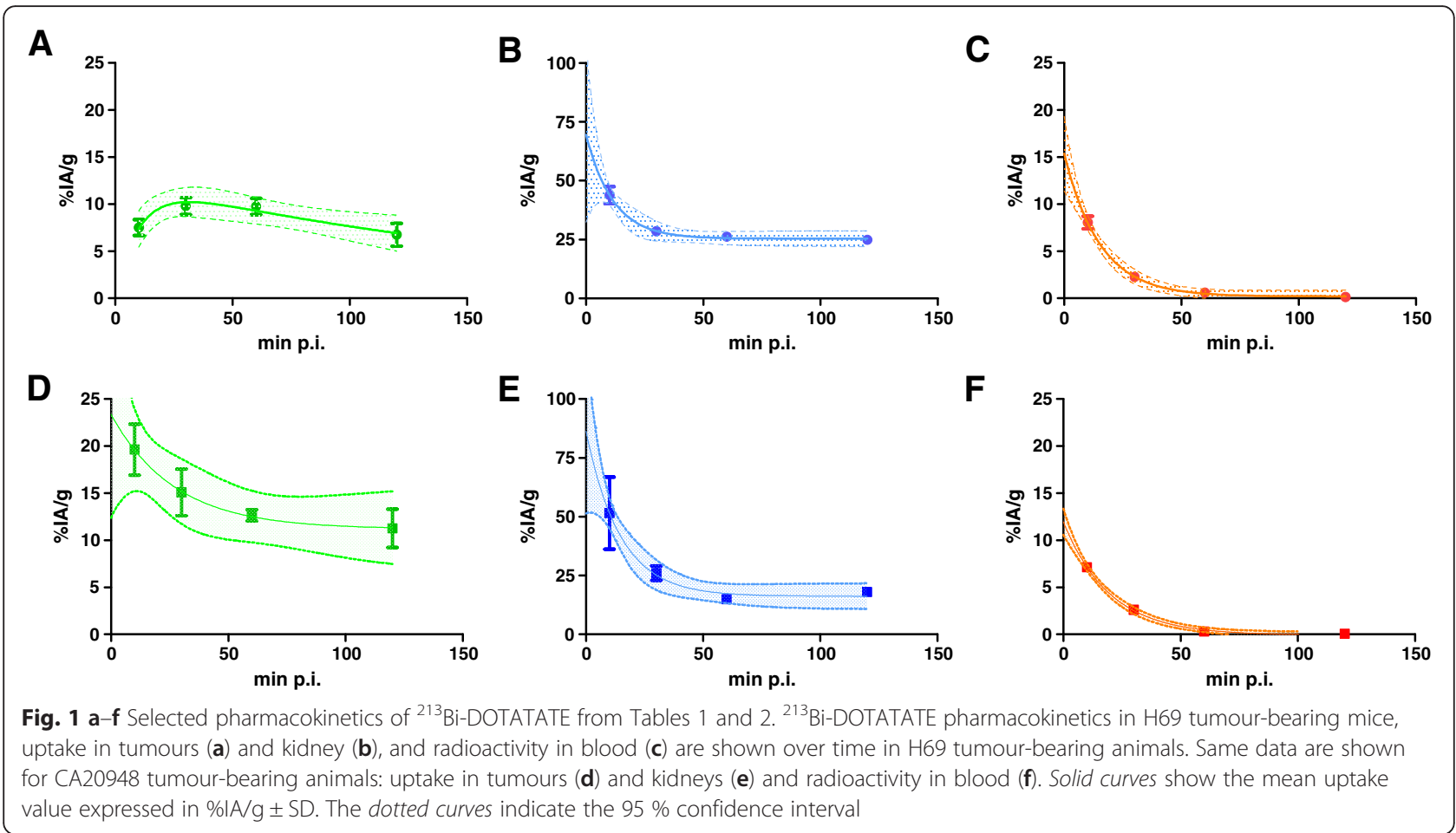

$$
D_{i}=\widetilde{A}_{i} \times S(i \leftarrow i)
$$

with $\tilde{A}_{i}$ the time-integrated activity (TIA) in organ $i$ and $S(i \leftarrow i)$ the absorbed dose rate per unit activity ( $S$ value). Only the self-dose $S$-values were considered and were obtained by interpolation between the sphere $S$ values for the measured organs or tumour mass. For small tumours, a minimum tumour mass of $50 \mathrm{mg}$ was used for the absorbed dose calculation. The $\tilde{A}_{i}$ in each organ was determined for ${ }^{213} \mathrm{Bi}$ and its daughters taking the biodistribution into account according to the methods shown in Additional file 1. The absorbed dose to the kidneys was calculated both with the spherical $S$ values and with the nephron model by Hobbs et al. [21]. Three hypothetical activity distributions were considered for this heterogeneous model: (1) homogeneous in the cortex, (2) proximal tubular cell uptake and (3) glomerular cell uptake.

\section{Statistics}

Statistical analysis was performed using $t$ test and using the nonparametric Mann-Whitney test. $P$ values $<0.05$ were considered statistically significant. For statistical significances in tumour growth patterns, one-way ANOVA was used. Values are presented as the mean and the standard deviation (SD).

\section{Results}

An incorporation yield of $\geq 99 \%$ and a RCP $\geq 85 \%$ were found for the radiopeptide, as determined by ITLC and HPLC, respectively.

\section{Pharmacokinetic studies}

Biodistribution studies using ${ }^{213} \mathrm{Bi}$-DOTATATE in H69 tumour-bearing mice revealed relatively high tumour uptake. The highest tumour uptake was reached at $30 \mathrm{~min}$ p.i., $9.8 \pm 2.4 \% \mathrm{IA} / \mathrm{g}$. Tumour uptake $T_{1 / 2}$ was $7 \pm 2 \mathrm{~min}$, and tumour clearance $T_{1 / 2}$ was $2.3 \pm 1.1 \mathrm{~h}$. An even higher

Table 1 Pharmacokinetics of ${ }^{213}$ Bi-DOTATATE at 10, 30, 60 and 120 min post injection (p.i.) in $\mathrm{H} 69$ tumour-bearing animals, expressed in mean $\% \mathrm{IA} / \mathrm{g} \pm \mathrm{SD}, n=7$

\begin{tabular}{lllll}
\hline Uptake $(\% \mathrm{IA} / \mathrm{g})$ & $10 \mathrm{~min}$ p.i. & $30 \mathrm{~min}$ p.i. & $60 \mathrm{~min}$ p.i. & $120 \mathrm{~min}$ p.i. \\
\hline Tumour & $7.5 \pm 2.2$ & $9.8 \pm 2.4$ & $9.8 \pm 2.3$ & $6.8 \pm 3.2$ \\
Muscle & $2.1 \pm 0.8$ & $1.1 \pm 0.2$ & $1.0 \pm 1.4$ & $0.5 \pm 0.4$ \\
Kidney & $44.0 \pm 9.6$ & $28.5 \pm 3.7$ & $26.2 \pm 2.6$ & $25.0 \pm 3.8$ \\
Pancreas & $3.2 \pm 0.3$ & $2.8 \pm 0.3$ & $1.8 \pm 0.2$ & $1.4 \pm 0.2$ \\
Liver & $2.1 \pm 0.8$ & $0.9 \pm 0.09$ & $0.4 \pm 0.06$ & $0.3 \pm 0.05$ \\
Blood & $8.1 \pm 1.8$ & $2.3 \pm 1.1$ & $0.6 \pm 0.2$ & $0.1 \pm 0.06$ \\
Spleen & $2.3 \pm 0.6$ & $1.0 \pm 0.09$ & $0.4 \pm 0.07$ & $0.2 \pm 0.07$ \\
Adrenal & $3.6 \pm 1.2$ & $2.0 \pm 0.6$ & $0.9 \pm 0.4$ & $0.7 \pm 0.4$ \\
Femur & $2.8 \pm 0.4$ & $1.5 \pm 0.4$ & $1.0 \pm 0.5$ & $0.4 \pm 0.4$ \\
\hline
\end{tabular}

$\% \mathrm{IA} / \mathrm{g}=$ mean percentage of total injected radioactivity per gram tissue 
Table 2 Pharmacokinetics of ${ }^{213}$ Bi-DOTATATE at 10, 30, 60 and 120 min post injection (p.i.) in CA20948 tumour-bearing animals, expressed in mean \%lA/g $\pm S D, n=7$

\begin{tabular}{lllll}
\hline Uptake $(\% \mid \mathrm{A} / \mathrm{g})$ & $10 \mathrm{~min}$ p.i. & $30 \mathrm{~min}$ p.i. & $60 \mathrm{~min}$ p.i. & $120 \mathrm{~min}$ p.i. \\
\hline Tumour & $19.6 \pm 6.6$ & $15.1 \pm 6.6$ & $12.7 \pm 1.6$ & $11.2 \pm 5.4$ \\
Muscle & $3.1 \pm 0.8$ & $1.5 \pm 0.8$ & $1.4 \pm 1.9$ & $0.2 \pm 0.2$ \\
Kidney & $51.5 \pm 16.6$ & $26.0 \pm 3.3$ & $15.0 \pm 1.5$ & $18.0 \pm 1.9$ \\
Pancreas & $3.6 \pm 0.2$ & $3.1 \pm 0.4$ & $1.5 \pm 0.3$ & $1.2 \pm 0.2$ \\
Liver & $2.0 \pm 0.2$ & $1.0 \pm 0.1$ & $0.2 \pm 0.04$ & $0.2 \pm 0.03$ \\
Blood & $7.1 \pm 1.0$ & $2.6 \pm 0.4$ & $0.3 \pm 0.2$ & $0.07 \pm 0.04$ \\
Spleen & $2.0 \pm 0.2$ & $1.1 \pm 0.4$ & $0.2 \pm 0.05$ & $0.2 \pm 0.04$ \\
Adrenal & $3.4 \pm 0.7$ & $2.2 \pm 0.8$ & $0.6 \pm 0.1$ & $0.4 \pm 0.1$ \\
Femur & $2.4 \pm 0.2$ & $1.4 \pm 0.2$ & $0.6 \pm 0.1$ & $0.2 \pm 0.04$ \\
\hline
\end{tabular}

$\% \mathrm{IA} / \mathrm{g}=$ mean percentage of total injected radioactivity per gram tissue renal activity compared to tumour uptake was found at all time points, the highest renal activity being found at $10 \mathrm{~min}$ p.i., $44 \pm 10 \% \mathrm{IA} / \mathrm{g}$. At $120 \mathrm{~min}$ p.i., the renal activity still was $25 \pm 3 \% \mathrm{IA} / \mathrm{g}$. Blood clearance in H69 tumourbearing animals was fast, and clearance $T_{1 / 2}$ was $11 \pm$ 2 min, see Fig. 1a-c. All other organs had relative low uptake at 120 min p.i., < $2 \% \mathrm{IA} / \mathrm{g}$, see Table 1 .

Tumour uptake of ${ }^{213} \mathrm{Bi}$-DOTATATE in CA20948 tumour-bearing mice was already highest at $10 \mathrm{~min}$ p.i., $19.6 \pm 6.6 \% \mathrm{IA} / \mathrm{g}$, and the tumour uptake $T_{1 / 2}$ was therefore unable to estimate. A clearance $T_{1 / 2}$ of $2.2 \pm$ $0.9 \mathrm{~h}$ was found for CA20948 tumours. Similar renal activity was found in CA20948 tumour-bearing animals. Renal activity was highest at $10 \mathrm{~min}$ p.i., $52 \pm$ $17 \% \mathrm{IA} / \mathrm{g}$. Renal clearance $T_{1 / 2}$ was $10 \pm 3 \mathrm{~min}$, whereas blood clearance $T_{1 / 2}$ was $14 \pm 1 \mathrm{~min}$, see Fig. 1d-f. At $120 \mathrm{~min}$ p.i., uptakes in other organs remained low, $<2 \% \mathrm{IA} / \mathrm{g}$, see Table 2 .

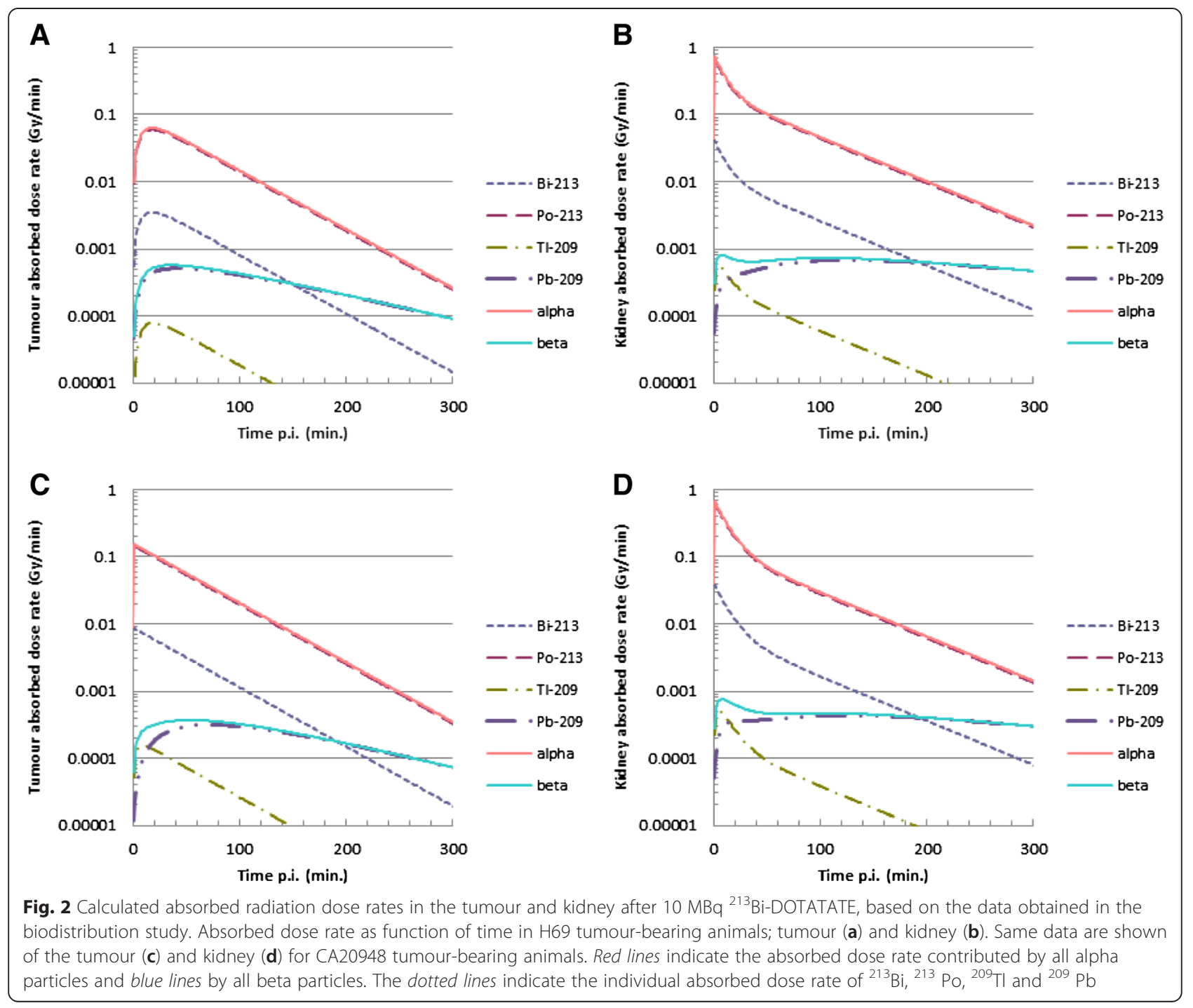




\section{Dosimetry}

Dosimetry calculations in both tumour models were based on the data obtained from the biodistribution studies at 10, 30, 60 and 120 min p.i. In H69 tumour-bearing mice, a relatively high tumourabsorbed radiation dose was estimated: $0.45 \mathrm{~Gy} / \mathrm{MBq}$ injected ${ }^{213}$ Bi-DOTATATE calculated for $100 \mathrm{mg}$ tumour. The renal-absorbed dose was four times as high as the tumour-absorbed radiation dose: $2 \mathrm{~Gy} / \mathrm{MBq}$ injected ${ }^{213} \mathrm{Bi}$-DOTATATE. The absorbed radiation dose of ${ }^{213} \mathrm{Bi}$-DOTATATE in the pancreas, spleen and liver was low compared to those in the tumour and kidney, 0.15 , 0.06 and $0.06 \mathrm{~Gy} / \mathrm{MBq}$, respectively. Similar results were observed in CA20948 tumour-bearing animals. A lower tumour-absorbed radiation dose was found than that in the kidneys: $0.78 \mathrm{~Gy} / \mathrm{MBq}$ in the tumour vs $1.6 \mathrm{~Gy} / \mathrm{MBq}$. Absorbed radiation doses in the pancreas, spleen and liver were $0.13,0.05$ and $0.06 \mathrm{~Gy} / \mathrm{MBq}$, respectively. For both tumour-bearing animal models, an absorbed dose of $<0.02 \mathrm{~Gy} / \mathrm{MBq}$ was found in the bone marrow.

Tumour- and kidney-absorbed radiation doses in H69 or CA20948 tumour-bearing animals were mainly caused by the $\alpha$-particles emitted. The absorbed dose rate was higher in CA20948 than in H69 tumours, and the kidneyabsorbed radiation dose was similar; see Fig. 2a-d. For an instantaneous uptake in the H69 tumour, its absorbed dose would rise by $12 \%$ to $0.50 \mathrm{~Gy} / \mathrm{MBq}$, whereas the absorbed dose to the CA20948 tumour is less dependent on the uptake kinetics: the dose is lowered by $2 \%$ to
$0.77 \mathrm{~Gy} / \mathrm{MBq}$ when the same uptake kinetics as for the H69 tumour is assumed.

\section{Targeted alpha therapy}

Therapeutic anti-tumour effects were observed in all animals treated with ${ }^{213} \mathrm{Bi}$-DOTATATE, see Fig. 3 . The tumour volume doubling time was $16 \pm 6$ days for the H69 and $10 \pm 5$ days for the CA20948 tumours, without any difference between the growth rate of small tumours and large tumours. The mean times to reach the end volume of $2000 \mathrm{~mm}^{3}$ of the control and treated animals of both tumour models are indicated in Table 3. For small tumours, tumour sizes $<50 \mathrm{~mm}^{3}$ could not be measured accurately; therefore, $50 \mathrm{~mm}^{3}$ was used as the minimum tumour sizes in our calculation and this was in three cases of H69 tumour-bearing animals with a small tumour and in all cases of CA20948 tumour-bearing animals with a small tumour. Overall calculated cumulative tumourabsorbed dose in small tumours was higher than large tumours. In CA20948 tumours, the cumulative tumourabsorbed dose was higher than in H69 tumours.

Shrinkage of tumours was observed in H69 tumourbearing animals with large tumours and a delay of tumour growth around 70 days after ${ }^{213} \mathrm{Bi}$-DOTATATE (cumulative injected activity of $9.4 \pm 0.7 \mathrm{MBq}$ ) treatment. In CA20948 tumour-bearing animals with large tumours, the inhibition of tumour growth was initiated around 30 days after therapy (cumulative injected activity 10.2 $\pm 0.4 \mathrm{MBq}$ ${ }^{213} \mathrm{Bi}$-DOTATATE), leading to a tumour growth delay
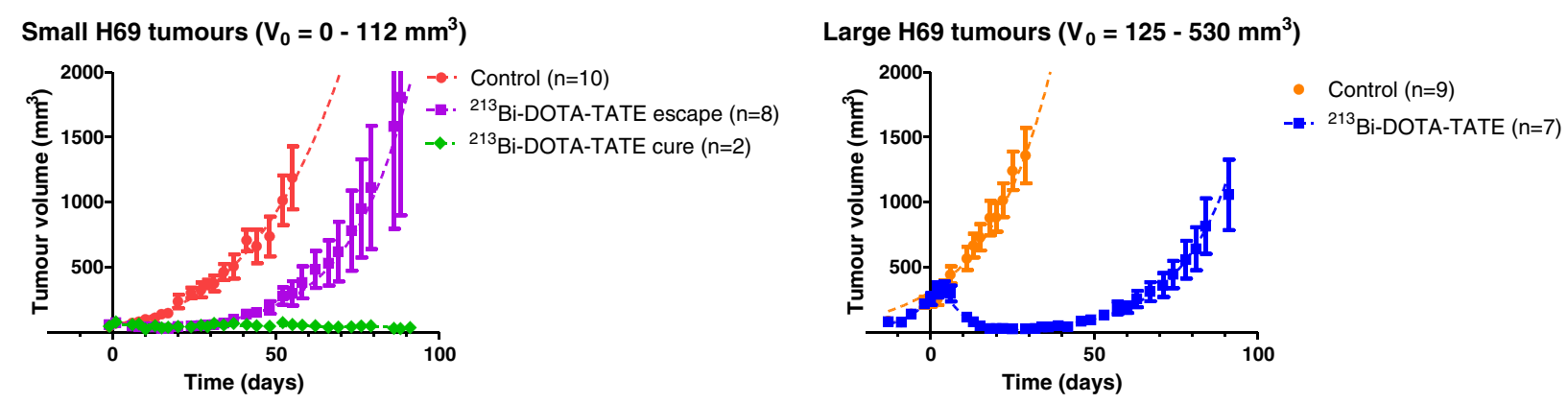

Small CA20948 tumours $\left(\mathrm{V}_{0}=0-39 \mathrm{~mm}^{3}\right)$
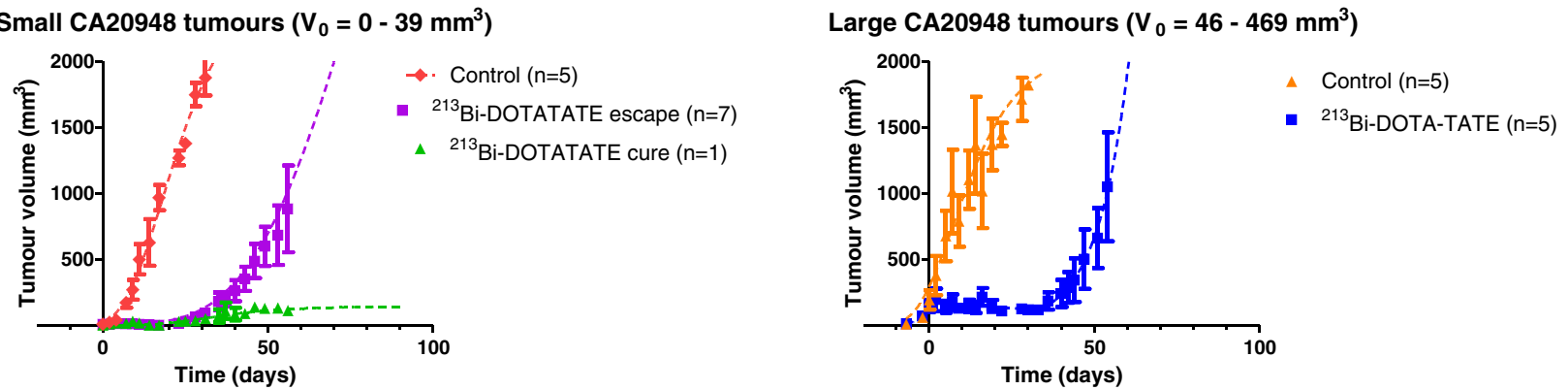

Fig. 3 Overview of mean tumour volumes \pm standard deviation (SD) in $\mathrm{mm}^{3}$ per group after treatment of ${ }^{213}$ Bi-DOTATATE (2-4 MBq per injection) or unlabelled DOTATATE (control) in $\mathrm{H} 69$ and CA20948 tumour-bearing mice for three consecutive days. $V_{0}$ is the starting tumour volume. The dotted curves show the extrapolated growth curves of the tumours assuming $100 \%$ survival of animals 
Table 3 An overview of mean time (days $=$ d) to reach a tumour volume of $2000 \mathrm{~mm}^{3}$ after ${ }^{213}$ Bi-DOTATATE therapy

\begin{tabular}{lllll}
\hline & H69 small & H69 large & CA20948 small & CA20948 large \\
\hline Control & $73 \pm 19 \mathrm{~d}$ & $38 \pm 11 \mathrm{~d}$ & $43 \pm 20 \mathrm{~d}$ & $27 \pm 14 \mathrm{~d}$ \\
Therapy & $102 \pm 19 \mathrm{~d}$ & $110 \pm 19 \mathrm{~d}$ & $76 \pm 15 \mathrm{~d}$ & $77 \pm 25 \mathrm{~d}$ \\
Tumour dose (Gy) & $5 \pm 1 \mathrm{~Gy}$ & $2.0 \pm 0.6 \mathrm{~Gy}$ & $17 \pm 1 \mathrm{~Gy}$ & $10 \pm 5 \mathrm{~Gy}$ \\
Growth delay (control vs therapy) & $28 \pm 9 \mathrm{~d}^{* * *}$ & $73 \pm 24 \mathrm{~d}^{*}$ & $33 \pm 17 \mathrm{~d}$ NS & $50 \pm 13 \mathrm{~d}^{* *}$ \\
\hline
\end{tabular}

NS not significant

${ }^{*}=P$ value $\leq 0.05 ;{ }^{* *}=P$ value $\leq 0.01 ; * * *=P$ value $\leq 0.001$

The tumour dose (Gy) \pm SD was estimated based on the tumour size at $T=0$ of therapy and the injected activity (MBq). Growth delay (days) was the $\Delta T$ for tumour to reach $2000 \mathrm{~mm}^{3}$ of therapy group vs control group

time of 50 days. Similar results were found in CA20948 tumour-bearing animals with small tumours after therapy (cumulative injected activity $7.7 \pm 0.4 \mathrm{MBq}$ ); a delay in tumour growth of around 30 days was observed, and in one of the animals, no tumour regrowth was observed. The delay time was estimated excluding the animals with cure or stable disease. In H69 tumour-bearing animals with small tumours, a delay of tumour growth was found for around 30 days after therapy (cumulative injected activity $7.4 \pm 0.5 \mathrm{MBq}$ ), and in two animals, no tumour regrowth was observed until the end of the study at day 90. Here, the two animals with cure/stable disease were excluded from the estimated delay time.

\section{Survival}

All animals treated with ${ }^{213} \mathrm{Bi}$-DOTATATE showed a higher survival rate than the control animals, see Fig. 4. In the control, non-TAT H69 tumour-bearing animals, a median survival of 34 days was found for animals bearing large tumours and 81 days for animals bearing small tumours. H69 tumour-bearing animals with small tumours had a median survival of $>90$ days after ${ }^{213} \mathrm{Bi}$-DOTATATE therapy (control vs treated, $P$ value $=<0.0001)$. Similar results were obtained in H69 tumour-bearing animals with large tumours after TAT; a median survival $>90$ days was found (control vs treated, $P$ value $=0.0195$ ). In the CA20948 tumourbearing animals without TAT, a median survival of 29 days was found for animals bearing large tumours, whereas for animals bearing small tumours, this was
35 days. After ${ }^{213} \mathrm{Bi}$-DOTATATE, a median survival of 70 days was found in the large-tumour-animal group (control vs treated, $P$ value $=$ not significant) and $>90$ days in the small-tumour-animal group (control vs treated, $P$ value $=0.005$ ).

\section{${ }^{99 m}$ Tc-DMSA uptake in the kidney}

Renal function was investigated in H69 and CA20948 tumour-bearing animals at day 90 after ${ }^{213} \mathrm{Bi}$-DOTATATE therapy or unlabelled DOTATATE treatment. Renal function was determined by renal uptake of ${ }^{99} \mathrm{~m}$ Tc-DMSA (25 MBq). No significant differences in ${ }^{99} \mathrm{~m}$ Tc-DMSA uptake in the kidneys were observed in ${ }^{213} \mathrm{Bi}$-DOTATATEtreated mice and control animals for both tumour models. In H69 tumour-bearing mice, a mean renal uptake of 2.6 $\pm 0.8 \mathrm{MBq}$ (mean concentration of $2.9 \pm 0.6 \mathrm{kBq} / \mathrm{mm}^{3}$ ) in the control group $(n=4)$ vs $2.1 \pm 0.9 \mathrm{MBq}$ (mean concentration of $\left.2.9 \pm 0.8 \mathrm{kBq} / \mathrm{mm}^{3}\right)$ in ${ }^{213} \mathrm{Bi}$-DOTATATEtreated animals $(n=8)$ was found. Similar results were obtained in CA20948 tumour-bearing animals: mean uptake of $2.1 \pm 0.2 \mathrm{MBq}$ (mean concentration of $3.1 \pm 0.4 \mathrm{kBq} /$ $\left.\mathrm{mm}^{3}\right)$ in ${ }^{213} \mathrm{Bi}$-DOTATATE-treated animals $(n=4)$ and $2.1 \pm 0.3 \mathrm{MBq}$ (mean concentration $3.2 \pm 0.3 \mathrm{kBq} / \mathrm{mm}^{3}$ ) in control $(n=2)$ were found. Figure $5 \mathrm{a}-\mathrm{e}$ shows ${ }^{99 \mathrm{~m}} \mathrm{Tc}-$ DMSA uptake in kidneys of both tumour models.

\section{Discussion}

In this study, we compared the efficacy of ${ }^{213} \mathrm{Bi}$-DOTATATE TAT in tumours of different sizes in two different tumour models, both with expression of $\mathrm{SSTR}_{2} .{ }^{213} \mathrm{Bi}$ -
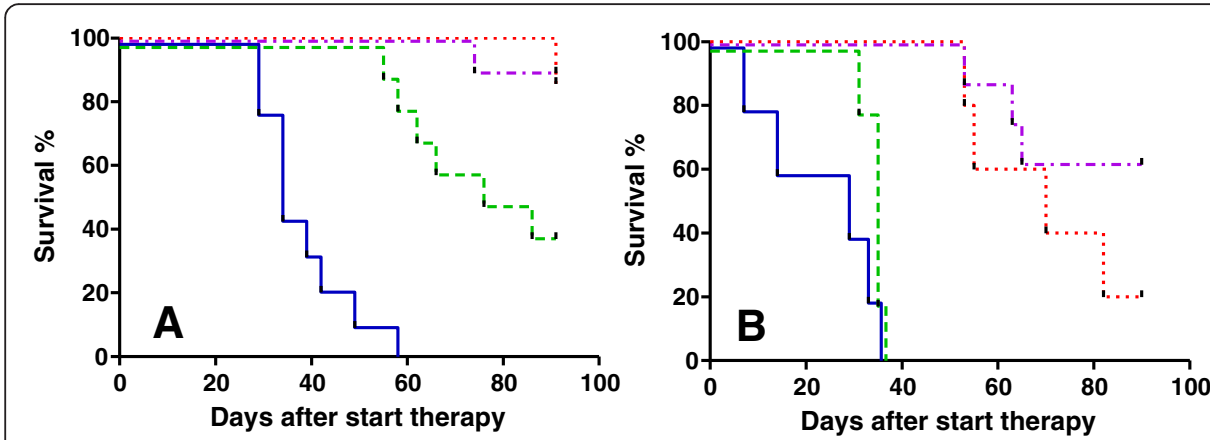

- Placebo: big tumor

.... . ${ }^{213} \mathrm{Bi}$-DOTA-TATE - big tumor

-ᄂ. Placebo: small tumor

.. ${ }^{213} \mathrm{Bi}$-DOTA-TATE - small tumor

Fig. 4 Survival (percentage) after ${ }^{213}$ Bi-DOTATATE or DOTATATE treatment in a H69 tumour-bearing and b CA20948 tumour-bearing mice 

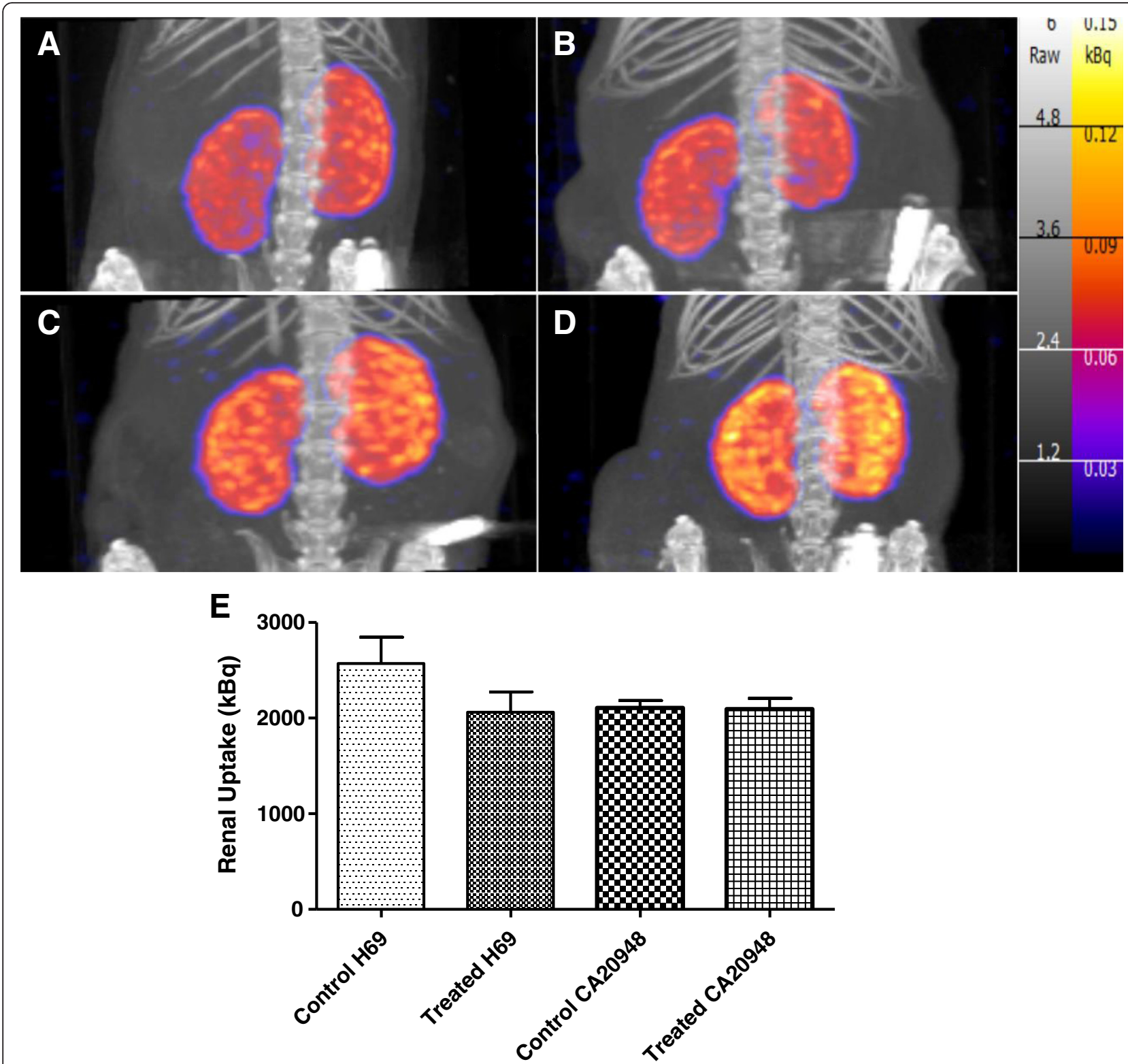

Fig. 5 a-d ${ }^{99 m}$ Tc-DMSA renal SPECT images in H69 control (a), ${ }^{213}$ Bi-DOTATATE-treated H69 mice (b), CA20948 control (c) and ${ }^{213}$ Bi-DOTATATE-treated CA20948 mice (d). e Renal uptake (in kBq) of ${ }^{99 \mathrm{~m} T c-D M S A}$ (25 MBq) in $\mathrm{H} 69$ and CA20948 tumour-bearing mice after therapy with ${ }^{213} \mathrm{Bi}-\mathrm{DOTATATE}$ or DOTATATE

DOTATATE showed a very good therapeutic effect in both small and large tumours in the two different tumour models. Tumour-absorbed doses (Gy) in CA20948 tumour-bearing animals were higher compared to those in H69 tumour-bearing mice. Despite these findings, ${ }^{213} \mathrm{Bi}$-DOTATATE showed greater therapeutic effects in H69 tumour-bearing animals than in CA20948 tumourbearing animals; a higher survival rate was observed in H69 tumour-bearing animals at the end of the study. However, no significant difference in tumour (re)growth inhibition time between the two tumour models was found, even though the absorbed dose in the tumour was higher in CA20948 tumour-bearing animals. In vitro, CA20948 cells appeared also more radioresistant than H69 cells; at external beam irradiation with 2 Gy, a survival fraction of H69 was 37 \% [13] and for CA20498, $52 \%$ was found [12].

In this study, we observed a higher anti-tumour effect in small tumours. In H69 tumour-bearing mice with small tumours, two animals showed stable disease and in eight animals, a partial response was found after treatment. In the case of CA20948 tumour-bearing animals with small tumours, one animal showed stable disease till the end of the study and partial response was found 
in eight of the animals after ${ }^{213} \mathrm{Bi}$-DOTATATE therapy. In H69 and CA20948 tumour-bearing animals with large tumours, a partial response was found. A longer tumour growth delay was found in animals with large tumours of both tumour models compared to small tumours. The time a tumour takes to reach $2000 \mathrm{~mm}^{3}$ in small tumours is longer than in large tumours; therefore, $\Delta$ delay time for tumour growth was used. Nevertheless, in animals showing regrowth, the small tumours were less responsive than the large tumours in both models.

The reason for partial remission can be explained as the distribution of the radiation-absorbed dose depends on the tumour size, the physical properties of the radionuclide and its possible inhomogeneous intratumoural distribution. In those cases where the tumour dimensions are smaller than the range of the ionizing particles, a large proportion of the absorbed energy can "escape", resulting in a suboptimal effect of ${ }^{213} \mathrm{Bi}$-DOTATATE TAT [22]. In larger tumours, also pronounced therapeutic effects were observed after ${ }^{213} \mathrm{Bi}$-DOTATATE therapies in both tumour models. However, no complete remissions were observed in these animals. Besides tumour perfusion [23] and inhomogeneous distribution of SSTR 2 , tumour size [17, 22], vessel density, permeability and tumour growth doubling time can play a critical role for the success of PRRT; this is especially true for $\alpha$ emitters, where the penetration depth in tissue is relatively short $(50-100 \mu \mathrm{m})$. In large tumours, often heterogeneity of receptor distribution [24] is observed, resulting in insufficient efficacy of the treatment and leading to probability survival of tumour cells. Therefore multiple fractions of therapy might improve the effect in larger tumours [25], especially in the case of TAT.

For somatostatin receptor-targeted PRRT, nephrotoxicity is often the dose-limiting factor, since reabsorption of radiolabelled somatostatin analogues by cells in the proximal tubule of the nephron occurs [26].

In this study, the calculated renal-absorbed dose using our simplified model was compared to the model described by Hobbs et al. [21]. We assumed all renal uptakes to be localized in the proximal tubular cells and took the glomerular cells as target. The absorbed dose in the glomeruli was $57 \%$ lower than for the mean absorbed kidney dose. On the other hand, the dose to the glomeruli is almost a factor 20 higher when all activity was concentrated in the glomerular cells. The homogeneous mean absorbed dose we calculated with the sphere model was $24 \%$ higher than when calculated with the cortex model from Hobbs et al. [21].

${ }^{99} \mathrm{~m}$ Tc-DSMA can provide information on renal function after renal tubular damage and has been used as renal marker in PRRT [27]. In this study, we did not find any changes in renal function in animals after TAT (highest cumulative activity used was $11.5 \mathrm{MBq}{ }^{213} \mathrm{Bi}$ -
DOTATATE). Kidney histology was performed in CA20948-bearing animals (data not shown), and no significant differences were observed in control vs treated animals. However, ${ }^{213} \mathrm{Bi}$-DOTATATE is known to accumulate in the kidney, leading to a high renal-absorbed dose. In patients, the maximum tolerated dose in kidney is postulated to be $23-28$ Gy [28-30]. Kidney protectants during TAT are therefore advised [9, 31, 32], especially when higher treatment activities are used. In another study, we found a maximum tolerated activity of 21.2 $\pm 1.7 \mathrm{MBq}$ in the presence of i.p. L-lysine as kidney protectant prior to the ${ }^{213} \mathrm{Bi}$-DOTATATE treatment; in the absence of L-lysine, the maximum tolerated activity was $<13.1 \pm 1.2 \mathrm{MBq}$ (manuscript in preparation).

Even though we have not been able to achieve complete remission of all tumours, this study demonstrated that TAT can be effective for the treatment of both large and small tumours. These findings are in line with those of Kratochwil et al. [8] who demonstrated the effectiveness of TAT in eight patients refractory to PRRT with ${ }^{177} \mathrm{Lu}$ and ${ }^{90} \mathrm{Y}$. We therefore believe that TAT is most promising for the treatment of NET. In combination with PRRT using ${ }^{90} \mathrm{Y}$ and ${ }^{177} \mathrm{Lu}$ peptides [33, 34], even greater therapeutic efficacy might be achieved, especially for in the treatment of patients with larger NET.

\section{Conclusions}

TAT with ${ }^{213}$ Bi-DOTATATE shows very good therapeutic efficacy for the treatment of both large and small tumours in these mouse models. In small tumours, ${ }^{213} \mathrm{Bi}$-DOTATATE demonstrated greater anti-tumour effect by its higher probability for inducing stable disease. Furthermore, TAT using ${ }^{213}$ Bi-DOTATATE demonstrated to be safe in our experimental setting, with kidneys as dose-limiting organs. ${ }^{213} \mathrm{Bi}$-DOTATATE therefore held great promise for targeted therapy for neuroendocrine tumours that express $\mathrm{SSTR}_{2}$.

\section{Ethics approval}

All applicable international, national and/or institutional guidelines for the care and use of animals were followed.

\section{Additional file}

\section{Additional file 1: Biodistribution of ${ }^{213} \mathrm{Bi}$ activity and its daughters} for single compartment kinetics.

\section{Competing interests}

Author Ho Sze Chan declares that she has no conflict of interest. Author Mark W. Konijnenberg declares that he has no conflict of interest. Author Erik de Blois declares that he has no conflict of interest. Author Stuart Koelewijn declares that he has no conflict of interest. Author Richard P. Baum declares that he has no conflict of interest. Author Alfred Morgenstern declares that he has no conflict of interest. Author Frank Bruchertseifer declares that he has no conflict of interest. Author Wouter A. Breeman owns stock in AAA. 
Author Marion de Jong is the chair of the Translational Molecular Imaging and Therapy Committee of the EANM and owns stock in AAA.

\section{Authors' contributions}

All authors read and approved the final manuscript.

\section{Funding}

This study was funded by DINSE Stiftung.

\section{Author details}

${ }^{1}$ Department of Nuclear Medicine, Erasmus Medical Center, Rotterdam, The Netherlands. ${ }^{2}$ Department of Radiology, Erasmus Medical Center, Rotterdam, The Netherlands. ${ }^{3}$ Institute for Transuranium Elements (ITU), Joint Research Centre, European Commission, Karlsruhe, Germany. ${ }^{4}$ Department of Nuclear Medicine/Center for PET/CT, Zentralklinik, Bad Berka, Germany.

\section{Received: 16 October 2015 Accepted: 6 January 2016} Published online: 20 January 2016

\section{References}

1. Bodei L, Kidd M, Baum RP, Modlin IM. PRRT: defining the paradigm shift to achieve standardization and individualization. J Nucl Med. 2014;55(11):1753-6. Epub 2014/09/27.

2. van der Zwan WA, Bodei L, Mueller-Brand J, de Herder WW, Kvols LK Kwekkeboom DJ. Gepnets update: radionuclide therapy in neuroendocrine tumors. Eur J Endocrinol. 2015;172(1):R1-8. Epub 2014/08/15.

3. Mulford DA, Scheinberg DA, Jurcic JG. The promise of targeted \{alpha\}particle therapy. J Nucl Med. 2005;46 Suppl 1:199S-204. Epub 2005/01/18.

4. Morgenstern A, Bruchertseifer F, Apostolidis C. Targeted alpha therapy with 213Bi. Curr Radiopharm. 2011;4(4):295-305. Epub 2011/12/29.

5. Jurcic JG, Caron PC, Nikula TK, Papadopoulos EB, Finn RD, Gansow OA, et al. Radiolabeled anti-CD33 monoclonal antibody M195 for myeloid leukemias. Cancer Res. 1995:55(23 Suppl):5908s-10. Epub 1995/12/01.

6. Norenberg JP, Krenning BJ, Konings IR, Kusewitt DF, Nayak TK, Anderson TL, et al. 213Bi-[DOTA0, Tyr3]octreotide peptide receptor radionuclide therapy of pancreatic tumors in a preclinical animal model. Clin Cancer Res. 2006; 12(3 Pt 1):897-903. Epub 2006/02/10.

7. Wild D, Frischknecht M, Zhang H, Morgenstern A, Bruchertseifer F, Boisclair J, et al. Alpha- versus beta-particle radiopeptide therapy in a human prostate cancer model (213Bi-DOTA-PESIN and 213Bi-AMBA versus 177LuDOTA-PESIN). Cancer Res. 2011;71(3):1009-18. Epub 2011/01/20.

8. Kratochwil C, Giesel FL, Bruchertseifer F, Mier W, Apostolidis C, Boll R, et al. (2)(1)(3)Bi-DOTATOC receptor-targeted alpha-radionuclide therapy induces remission in neuroendocrine tumours refractory to beta radiation: a first-inhuman experience. Eur J Nucl Med Mol Imaging. 2014;41(11):2106-19. Epub 2014/07/30.

9. Melis M, Bijster M, de Visser M, Konijnenberg MW, de Swart J, Rolleman EJ, et al. Dose-response effect of Gelofusine on renal uptake and retention of radiolabelled octreotate in rats with CA20948 tumours. Eur J Nucl Med Mol Imaging. 2009;36(12):1968-76. Epub 2009/07/09.

10. de Jong M, Breeman WA, Bernard BF, Bakker WH, Visser TJ, Kooij PP, et al. Tumor response after [(90)Y-DOTA(0), Tyr(3)]octreotide radionuclide therapy in a transplantable rat tumor model is dependent on tumor size. J Nucl Med. 2001;42(12):1841-6. Epub 2001/12/26.

11. Bison SM, Pool SE, Koelewijn SJ, van der Graaf LM, Groen HC, Melis M, et al. Peptide receptor radionuclide therapy (PRRT) with [(177)Lu-DOTA(0), $\operatorname{Tyr}(3)$ ]octreotate in combination with RAD001 treatment: further investigations on tumor metastasis and response in the rat pancreatic CA20948 tumor model. EJNMMI Res. 2014:4:21. Epub 2014/07/06.

12. Verwijnen S, Capello A, Bernard B, van den Aardweg G, Konijnenberg M, Breeman W, et al. Low-dose-rate irradiation by 131 I versus high-dose-rate external-beam irradiation in the rat pancreatic tumor cell line CA20948. Cancer Biother Radiopharm. 2004;19(3):285-92. Epub 2004/08/03.

13. Bjork-Eriksson T, West C, Karlsson E, Mercke C. Discrimination of human tumor radioresponsiveness using low-dose rate irradiation. Int J Radiat Oncol Biol Phys. 1998:42(5):1147-53. Epub 1998/12/30.

14. McDevitt MR, Finn RD, Sgouros G, Ma D, Scheinberg DA. An 225Ac/213Bi generator system for therapeutic clinical applications: construction and operation. Appl Radiat Isot. 1999;50(5):895-904. Epub 1999/04/24.

15. de Blois E, Sze Chan H, Naidoo C, Prince D, Krenning EP, Breeman WA. Characteristics of $\mathrm{SnO} 2$-based $68 \mathrm{Ge} / 68 \mathrm{Ga}$ generator and aspects of radiolabelling DOTA-peptides. Appl Radiat Isot. 2011;69(2):308-15. Epub 2010/12/15

16. Forrer F, Rolleman E, Bijster M, Melis M, Bernard B, Krenning EP, et al. From outside to inside? Dose-dependent renal tubular damage after high-dose peptide receptor radionuclide therapy in rats measured with in vivo (99m)Tc-DMSA-SPECT and molecular imaging. Cancer Biother Radiopharm. 2007;22(1):40-9. Epub 2007/07/14.

17. Konijnenberg MW, Breeman WA, de Blois E, Chan HS, Boerman OC, Laverman $\mathrm{P}$, et al. Therapeutic application of CCK2R-targeting PP-F11: influence of particle range, activity and peptide amount. Eur J Nucl Med Mol Imaging Res. 2014:4:15.

18. MCNP- a general Monte Carlo N-particle transport code, version 5 vol. 1. Overview and theory [database on the Internet]. Los Alamos National Lab Report. 2003.

19. Hendricks JS. MCNPX extensions version 2.5.0. 2005.

20. Eckerman KEA. MIRD: radionuclide data and decay schemes. 2nd ed. Reston: Society of Nuclear Medicine and Molecular Imaging; 2008.

21. Hobbs RF, Song H, Huso DL, Sundel MH, Sgouros G. A nephron-based model of the kidneys for macro-to-micro alpha-particle dosimetry. Phys Med Biol. 2012;57(13):4403-24. Epub 2012/06/19.

22. O'Donoghue JA, Bardies M, Wheldon TE. Relationships between tumor size and curability for uniformly targeted therapy with beta-emitting radionuclides. J Nucl Med. 1995;36(10):1902-9. Epub 1995/10/01.

23. Gillies RJ, Schornack PA, Secomb TW, Raghunand N. Causes and effects of heterogeneous perfusion in tumors. Neoplasia. 1999;1(3):197-207. Epub 2000/08/10.

24. Bol K, Haeck JC, Groen HC, Niessen WJ, Bernsen MR, de Jong M, et al. Can DCE-MRI explain the heterogeneity in radiopeptide uptake imaged by SPECT in a pancreatic neuroendocrine tumor model? PLoS One. 2013;8(10): e77076. Epub 2013/10/12.

25. Pach D, Sowa-Staszczak A, Kunikowska J, Krolicki L, Trofimiuk M, Stefanska A, et al. Repeated cycles of peptide receptor radionuclide therapy (PRRT)results and side-effects of the radioisotope 90Y-DOTA TATE, 177LU-DOTA TATE or 90Y/177Lu-DOTA TATE therapy in patients with disseminated NET. Radiother Oncol. 2012;102(1):45-50. Epub 2011/09/03.

26. Valkema R, Pauwels SA, Kvols LK, Kwekkeboom DJ, Jamar F, de Jong M, et al. Long-term follow-up of renal function after peptide receptor radiation therapy with (90)Y-DOTA(0), Tyr(3)-octreotide and (177)Lu-DOTA(0), Tyr(3)octreotate. J Nucl Med. 2005;46 Suppl 1:83S-91. Epub 2005/01/18.

27. Weyer K, Nielsen R, Petersen SV, Christensen El, Rehling M, Birn H. Renal uptake of $99 \mathrm{mTc}$-dimercaptosuccinic acid is dependent on normal proximal tubule receptor-mediated endocytosis. J Nucl Med. 2013;54(1):159-65. Epub 2012/12/13

28. Dawson LA, Kavanagh BD, Paulino AC, Das SK, Miften M, Li XA, et al. Radiation-associated kidney injury. Int J Radiat Oncol Biol Phys. 2010;76(3 Suppl):S108-15. Epub 2010/03/05.

29. Bodei L, Cremonesi M, Ferrari M, Pacifici M, Grana CM, Bartolomei M, et al. Long-term evaluation of renal toxicity after peptide receptor radionuclide therapy with 90Y-DOTATOC and 177LU-DOTATATE: the role of associated risk factors. Eur J Nucl Med Mol Imaging. 2008;35(10): 1847-56. Epub 2008/04/23

30. Guerriero F, Ferrari ME, Botta F, Fioroni F, Grassi E, Versari A, et al. Kidney dosimetry in (1)(7)(7)Lu and (9)(0)Y peptide receptor radionuclide therapy: influence of image timing, time-activity integration method, and risk factors. Biomed Res Int. 2013:2013:935351. Epub 2013/07/19.

31. Bernard BF, Krenning EP, Breeman WA, Rolleman EJ, Bakker WH, Visser TJ, et al. D-lysine reduction of indium-111 octreotide and yttrium-90 octreotide renal uptake. J Nucl Med. 1997;38(12):1929-33. Epub 1998/01/16.

32. Kobayashi H, Yoo TM, Kim IS, Kim MK, Le N, Webber KO, et al. L-lysine effectively blocks renal uptake of I-125- or Tc-99m-labeled anti-Tac disulfide-stabilized Fv fragment. Cancer Res. 1996:56(16):3788-95.

33. de Jong M, Breeman WA, Valkema R, Bernard BF, Krenning EP. Combination radionuclide therapy using $177 \mathrm{Lu}$ - and $90 \mathrm{Y}$-labeled somatostatin analogs. J Nucl Med. 2005;46 Suppl 1:13S-7. Epub 2005/01/18

34. Walrand S, Hanin FX, Pauwels S, Jamar F. Tumour control probability derived from dose distribution in homogeneous and heterogeneous models: assuming similar pharmacokinetics, (125)Sn-(177)Lu is superior to (90)Y-(177)Lu in peptide receptor radiotherapy. Phys Med Biol. 2012;57(13): 4263-75. Epub 2012/06/19. 